\title{
Chemo-Mechanically Regulated Oscillation of an Enzymatic Reaction
}

\section{Citation}

He, Ximin, Ronn S. Friedlander, Lauren D. Zarzar, and Joanna Aizenberg. 2013. “ChemoMechanically Regulated Oscillation of an Enzymatic Reaction." Chemistry of Materials 25 (4) (February 11): 521-523. doi:10.1021/cm303313a.

\section{Published Version}

doi:10.1021/cm303313a

\section{Permanent link}

http://nrs.harvard.edu/urn-3:HUL.InstRepos:37255485

\section{Terms of Use}

This article was downloaded from Harvard University's DASH repository, and is made available under the terms and conditions applicable to Open Access Policy Articles, as set forth at http:// nrs.harvard.edu/urn-3:HUL.InstRepos:dash.current.terms-of-use\#OAP

\section{Share Your Story}

The Harvard community has made this article openly available.

Please share how this access benefits you. Submit a story.

Accessibility 


\title{
Chemo-Mechanically Regulated Oscillation of an Enzymatic Reaction
}

\author{
Ximin He, ${ }^{\dagger}$ Ronn S. Friedlander, ${ }^{\S}$ Lauren D. Zarzar, $"$ Joanna Aizenberg ${ }^{\star, \dagger, \ddagger}, \|$ \\ ${ }^{\dagger}$ School of Engineering and Applied Sciences, Harvard University, Cambridge, Massachusetts 02138, USA; ${ }^{\circledR}$ Harvard- \\ MIT Division of Health Sciences and Technology, Cambridge, Massachusetts 02139, USA; "Department of Chemistry \\ and Chemical Biology, Harvard University, Cambridge, Massachusetts 02138, USA; ${ }^{\ddagger}$ Kavli Institute for Bionano \\ Science and technology at Harvard University, Cambridge, Massachusetts 02138, USA
}

KEYWORDS. Responsive material, chemo-mechanical, oscillation, enzymatic reaction.

A necessity of all living organisms from prokaryotes to mammals is the capability to respond rapidly to a changing environment via a variety of adaptive mechanisms. At the core of this adaptive nature are hierarchical signaling cascades that allow the organism to transform a stimulus signal into a variety of outputs-often visible or otherwise detectable readouts (color change, movement, etc.). Essential to these functions are sophisticated receptors that freely transduce chemical and mechanical as well as other types of energy. These mechano-chemical interconversions occur at various length scales, from the nano-scale (e.g. the ribosome procession along an mRNA strand, F1-ATPase), ${ }^{2}$ to the micro-scale (e.g. flagellar motors, hair cells) ${ }^{3,4}$ and even the macro-scale (e.g. muscle movement by myocyte action). ${ }^{5}$

In contrast to nature's highly integrated and versatile cascades allowing transitions between biochemical and mechanical processes, our current synthetic world has few materials systems (e.g. responsive microfluidic valves) ${ }^{6}$ that integrate mechano-chemical $(\mathrm{M} \rightarrow \mathrm{C})$ transductions (e.g. sensors $)^{7,8}$ with chemo-mechanical $(\mathrm{C} \rightarrow \mathrm{M})$ ones (e.g. actuators $)^{9-12}$ thereby enabling coupling between these two types of energy with various outputs $\left(\mathrm{C}_{1} \rightarrow \mathrm{M} \rightarrow \mathrm{C}_{2} \rightarrow\right.$ output$){ }^{13,14} \quad$ More importantly, existing synthetic systems are generally incapable of hierarchically amplifying signals arising from the molecular level to give macroscopic readouts that are easily detectable, which is a highly desired property of next-generation materials ${ }^{15,16}$, as well as biosensing and analytical devices. ${ }^{17}$ Incorporating sophisticated biological process into materials and larger hierarchical systems presents a challenge and requires a broad-based design capable of mediating a large repertoire of biochemical processes.

The numerous biological examples of chemo-mechanical transduction and transformation show us that nature's bottom-up approach to the assembly of functional structures relies upon hierarchical ordering of molecular and cellular subunits through compartmentalization. ${ }^{18}$ It is via the coordinated activities of constituent parts that molecularscale events can trigger macroscale responses, such as the expansion and contraction of cephalopod chromatophores that determine the organism's overall apparent color pattern $^{19}$ and ATP-driven muscle contraction. ${ }^{5}$ Inspired by these and other similar phenomena, we have recently developed Self-regulated Mechano-chemical Adaptively Reconfigurable Tunable Systems (SMARTS), ${ }^{13}$ which offer smooth coupling and coordination of microscopic and macroscopic signals with fast mechanical action and a diverse range of chemical inputs and outputs. By embedding catalyst-bearing 'skeletal' microstructures into a $\mathrm{pH}$ - or temperature-responsive hydrogel 'muscle' and introducing a fluid containing reactive 'nutrients', we created a platform that allowed us to precisely control a number of externally regulated $C_{1} \rightarrow M \rightarrow C_{2}$ systems, such as pulsed gas generation and dynamic fluorescence quenching. Incorporation of carefully designed feedback mechanisms $(C \rightleftarrows M)$ into these systems has enabled continuous chemical, thermal and mechanical energy interconversions, resulting in autonomous, self-sustained dynamic materials.

Biochemical reactions, if integrated with the highly tunable and customizable SMARTS, would open doors to the control of innumerable sophisticated, environmentally friendly, enzymatic or other biological activities useful for self-regulating medical implants such as biomolecule detection, separation, and signal amplification. Biochemical processes in general are typically sensitive to $\mathrm{pH}$, temperature and salt concentrations, requiring a narrow range to operate successfully. Therefore, we aimed to tailor SMARTS to function in delicate, biologically-relevant environments in order for it to be ultimately capable of regulating complex, multi-component biochemical processes. This would greatly expand the scope of SMARTS and demonstrate the utility of this broad-based platform for biomedical applications and biological studies. Specifically, the exquisite controllability provided by SMARTS, which enables the transport of biomolecules between distinct aqueous environments via microstructure actuation, allows for externally regulated, oscillating biochemical reactions with programmable cycles and outputs. Here, we 


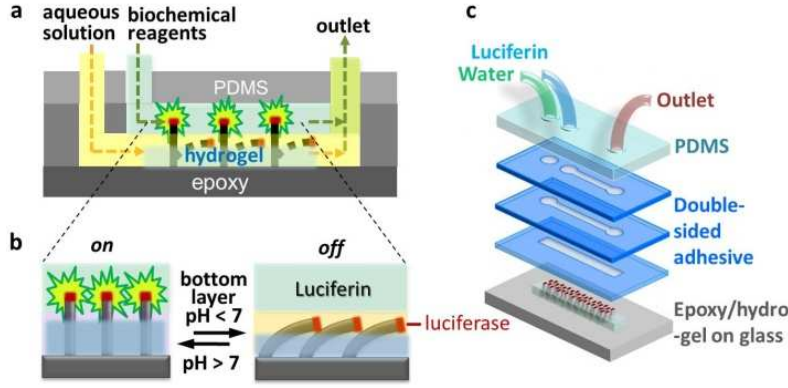

Figure 1. Fabrication of the oscillating bioluminescence reactor. a. Side-view schematic of oscillations in a bioluminescence-generating enzymatic reaction triggered by $\mathrm{pH}$, using a $\mathrm{pH}$-responsive gel to actuate epoxy microstructures immersed in an aqueous bilayer in a microfluidic device. b. Close-up schematic showing the reversible chemo-mechanical "on-off" switching of bioluminescence-generating luciferin oxidization by the enzyme LUC. c. Schematic of the fabrication of the microfluidic device that allows forming an aqueous bilayer. A polyacrylic double-sided adhesive tape stack with a rectangular channel and two vertically-separated inlets and one outlet was placed on top of the microfins and served as the walls of the channel, which was sealed by a PDMS sheet.

demonstrate chemo-mechanically mediated cyclic "on-off" switching of an exemplary enzymatic reaction-the luciferase (LUC)-catalyzed oxidation of luciferin using SMARTS. In this system, we convert $\mathrm{pH}$ changes to mechanical movement which in turn triggers a biochemical reaction that generates light. In this way we create a signal cascade/converter capable of translating signals at the nanoscale to outputs that can be visualized at the macro-scale.

Our system is composed of an array of epoxy microfins embedded in $\mathrm{pH}$-sensitive hydrogel and immersed in an aqueous bilayer formed in a microfluidic channel (Fig. 1a,b). To generate the microfins, silicon masters with a staggered array of microfins (each fin being $2 \mu \mathrm{m}$ wide, $10 \mu \mathrm{m}$ long and $18 \mu \mathrm{m}$ tall) was replicated in epoxy (9:1 (w/w) UVO-114 (Epoxy technology):glycidyl methacrylate) (see SI for details). To embed microfin structures into a hydrogel film, a drop of hydrogel precursor solution was placed on the freshlyprepared microfin surface and was cured under broadband UV illumination (See SI for details). Because of the instability of the enzyme LUC outside of the $\mathrm{pH}$ range of 6.0-9.0, we tuned the volume phase transition $\mathrm{pH}$ of the hydrogel poly(acrylamide-co-acrylic acid) ${ }^{20}$ from $\mathrm{pH} 4.3$ to $\mathrm{pH} 7$, by introducing $10 \%(\mathrm{w} / \mathrm{v})$ dodecyl acrylate co-monomer to the precursor $\mathrm{pH}$-responsive hydrogel solution. ${ }^{21}$

For the cyclic enzymatic reaction, fin tips were functionalized with LUC (Fig. S1). The localization of LUC to the fin tips allowed for movement of the catalyst between the two aqueous layers using the SMARTS system (Fig. 1 a,b). LUC catalyzes the oxidation of luciferin to generate light via the following reaction:

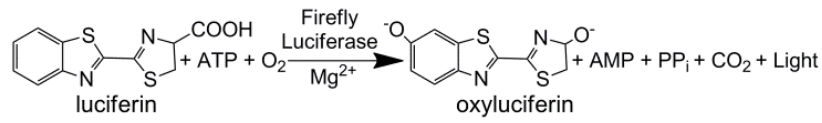

An aqueous solution of D-luciferin sodium salt, $\mathrm{MgCl}_{2}$ and ATP magnesium salt in HEPES $\mathrm{pH} 7.5$ buffer was used in the top fluid layer as the reagent medium. The reagent solution was oxygenated and the $\mathrm{O}_{2}$-permeable PDMS channel allowed further diffusion of $\mathrm{O}_{2}$ for the oxidation reaction.

To create a vertically-separated aqueous bilayer, a microfluidic channel with two inlets of different depths was fabricated on top of the hydrogel-embedded microfins (Fig. 1c). Reagent solution and water were flowed into the two inlets, creating a laminar flow interface that passed below the tips of the microfins. The bottom fluid was an alternating flow of $\mathrm{pH}$ 6.o sodium citrate and $\mathrm{pH} 9.0$ sodium tetraborate buffers to induce expansion and contraction of the hydrogel thereby driving the microfin actuation.
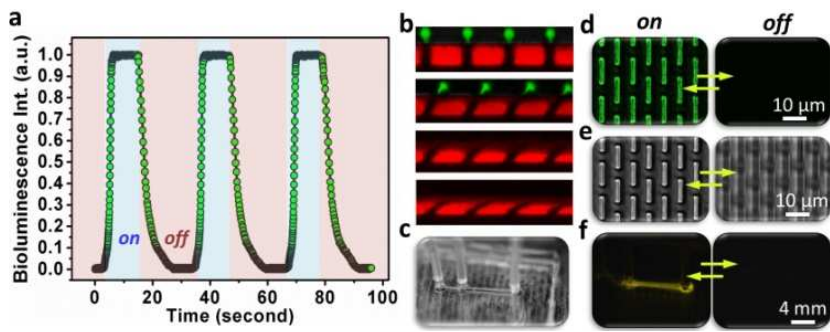

Figure 2. Dynamic light emission shown at the macro- and micro-scale by a mechanically-controlled bioluminescence reaction in SMARTS. a. Oscillations in bioluminescence intensity from controlled LUC-catalyzed luciferin oxidation synchronous with the oscillating movement of the LUCfunctionalized microfins driven by the expansion and contraction of the $\mathrm{pH}$-responsive gel resulting from alternating flow of the buffers at $\mathrm{pH} 6$ (pink) and 9 (blue) through the microfluidic device. $\mathrm{b}$. XZ sections of fins at various stages of actuation. Images are overlays of two emission channels, red: rhodamine-conjugated hydrogel; green: luminescent emission from the luciferin oxidation reaction. c. Digital camera photos of the microfluidic device. d. Confocal micrographs of microfins with luminescence on/off recorded without exogenous light. e. Transmitted light micrographs, revealing the configuration of bent microfins without bioluminescence. $f$. Digital camera photos of the microfluidic device without exogenous light, showing the macroscopic effect.

Microscopic fluorescence imaging and video recording were done using a confocal microscope equipped with an avalanche photodiode detector (APD) for quantification of the light emission. We simultaneously recorded the macroscopic dynamic processes while the microscopic videos and images were taken, as seen in Movie 1 (SI) and Fig. 2. The intensity of the reaction-generated luminescence was measured from each focal plane containing fin tips recorded with the APD. Fig. 2a shows the periodic change in the emitted light intensity during the microfin actuation; light intensity increases sharply as the fins stand upright with the flow of a $\mathrm{pH} 9$ buffer in the bottom layer, and the intensity quickly decreases as the tips bend down with the flow of $\mathrm{pH}$ 6 buffer in the bottom layer. We attribute the observed bioluminescence without the typical 'flash' behavior ${ }^{22}$ for the reaction to the flow system, which prevented the accumulation of oxyluciferin and dehydroluciferyl-adenylate and thus the inhibition of enzyme. ${ }^{23}$ The observed luminescence decay in the 'off' phase perhaps resulted from the residual oxyluciferin or a small quantity of luciferin remaining on the tips across the interface. The rapid responsiveness and precise synchronization of the light 
emission and the microfin movement in and out of the reagent layer during multiple cycles are well evidenced by the time-resolved bioluminescence intensity in Fig. 2a and Movie 1. Side-view configuration images of SMARTS using confocal XZT scanning and fluorescently labeled hydrogel (Fig. 2b) clearly reveal the dynamics of luminescence generation on fin tips during all stages of actuation (Fig. $\mathrm{S}_{3}$ ). The microscope images in Fig. 2d display the "on" and "off" states of light emission correlated to the upright or bent configurations of the fins. The microfin actuation was also recorded in transmission mode (Fig. 2e) to reveal the structures' configuration during the bent state (without light emission from bioluminescence). A series of control reactions conducted at $\mathrm{pH} \mathrm{6,7.5}$ and 9 in a static system (Fig. S2) emitted light without appreciable changes in intensity indicating that luminescence is not lost due to $\mathrm{pH}$ changes within the operating $\mathrm{pH}$ range. This confirmed that the disappearance of luminescence at $\mathrm{pH} 6$ resulted from the cessation of the reaction due to the tips bending away from top reagent layer rather than from the quenching of luminescence or dysfunction of the enzyme. The proper function of the enzyme is further supported by the sustained luminescence through 30-40 actuation cycles. Importantly, intermittent light emission was also apparent macroscopically: in the channel of the microfluidic device shown in Fig. 2c, visible readouts arising from the periodic bioluminescent reaction were detected (Fig. 2f).

The precise and swift on/off switching of the bioluminescent reaction (Fig. S3) demonstrates the excellent coordination of the chemically-induced mechanical motion $\left(\mathrm{C}_{1} \rightarrow \mathrm{M}\right)$ with the mechanically-modulated enzymatic activity $\left(\mathrm{M} \rightarrow \mathrm{C}_{2}\right)$. Using periodic changes in $\mathrm{pH}$ in the bottom layer as the stimulus, we realized a synchronous cascade of chemo-mechano-(bio)chemical light generation cycles. Through $\mathrm{pH}$-mediated swelling of the hydrogel "muscle", the coordinated bending of tens of thousands of catalystdecorated microfins generated light in the top fluid layer visible to the naked eye. The multi-scale cascade provides readouts of unrelated but coupled chemical events (i.e. $\mathrm{pH}$ change to light emission), and can be used to arbitrarily couple a vast range of reactions.

In summary, we have demonstrated precise control of biochemical signal transduction using a model system composed of LUC bound to micro-structure tips, catalyzing the oxidation of luciferin in an oscillatory fashion. The SMARTS device is shown to be both compatible with delicate biological constraints and capable of accommodating enzymatic reactions for signal transduction, attributed to its modularity, tunability, and physical simplicity. Moreover, the hybrid hydrogel-microstructured surface is well integrated in a microfluidic channel, showing that this chemo-mechanical system can be readily applied in microfluidic lab-on-a-chip systems to create effective, complex, and highly integrated microfluidic networks for automated biological processes. ${ }^{22}$ The potential variety of switchable biochemical reactions that could be accommodated by this $C_{1} \rightarrow M \rightarrow C_{2}$ cascade is complemented by the customizability of the hydrogel response, which can, in turn, be tailored to a wide range of stimuli, such as $\mathrm{pH}$, heat, light, glucose or other metabolic compounds, as well as the vast varieties of outputs, such as gas generation, color change, DNA polymerization, and proteolysis thus improving the combinatorial diversity of coupled effects. This work demonstrates an important step toward biomimetic, responsive materials, and should pave the way for more applications in microfluidic and biosensor systems, such as in-line sensors with novel readouts and outputs.

\section{ASSOCIATED CONTENT}

\section{Supporting Information}

I. Methods and Characterization; II. Control study of $\mathrm{pH}$ effect on the bioluminescence; a movie. This material is available free of charge via the Internet at http://pubs.acs.org.

\section{AUTHOR INFORMATION}

\section{Corresponding Author: jaiz@seas.harvard.edu}

\section{ACKNOWLEDGMENT}

The authors thank M. Aizenberg for valuable advice on this work and help with manuscript preparation, and A. Ehrlicher and $\mathrm{T}$. Kodger for technical assistance with luminescence imaging using confocal microscope. This work was supported by the US DOE under award DE-SCooo5247.

\section{REFERENCES}

(1)Prosser, B. L.; Ward, C. W.; Lederer, W. J. Sciencezo11, 333, 1440. (2)Sambongi, Y.; Iko, Y.; Tanabe, M.; Omote, H.; Iwamoto-Kihara, A.; Ueda, I.; Yanagida, T.; Wada, Y.; Futai, M. Science 1999, 286, 1722. (3)Berg, H. C. Annu. Rev. Biochem. 2003, 72, 19.

(4)Fettiplace, R.; Hackney, C. M. Nat. Rev. Neurosci. 2006, 7, 19.

(5)Hess, H. Annu. Rev. Biomed. Eng.2011, 13, 429.

(6)Beebe, D. J.; Moore, J. S.; Bauer, J. M.; Yu, Q.; Liu, R. H.; Devadoss, C.; Jo, B-H. Nature 200o, 404, 288.

(7)Ariga, K.; Mori, T.; Hill, J. P. Chem. Sci.2o11, 2, 195.

(8)Todres, Z. V. Organic mechanochemistry and its practical applications; CRC/Taylor \& Francis, 2006.

(9)Lahann, J.; Langer, R. MRS Bull.2005, 30, 185.

(10)He, X. M.; Li, C.; Chen, F. G.; Shi, G. Q. Adv. Funct. Mater. 2oo7, 17, 2911.

(11)Sidorenko, A.; Krupenkin, T.; Taylor, A.; Fratzl, P.; Aizenberg, J.

Science2007, 315, 487 .

(12)Paxton, W. F.; Sundararajan, S.; Mallouk, T. E.; Sen, A. Angew.

Chem. Int. Ed.20o6, 45, 5420.

(13)He, X., Aizenberg, M., Kuksenok, O., Zarzar, L.D., Shastri, A.,

Balazs, A.C., Aizenberg, J. Nature 2012, 487, 214.

(14)Fratzl, P.; Barth, F. G. Nature 2009, 462, 442.

(15)Stuart, M. A. C.; Huck, W. T. S.; Genzer, J.; Muller, M.; Ober, C.; Stamm, M.; Sukhorukov, G. B.; Szleifer, I.; Tsukruk, V. V.; Urban, M.; Winnik, F.; Zauscher, S.; Luzinov, I.; Minko, S. Nat. Mater.2010, 9, 101. (16)Kim, P.; Zarzar, L. D.; He, X. M.; Grinthal, A.; Aizenberg, J. Curr. Opin. Solid. St. M. 2011, 15, 236.

(17)Li, D. B.; Paxton, W. F.; Baughman, R. H.; Huang, T. J.; Stoddart, J. F.; Weiss, P. S. MRS Bull. 2009, 34, 671.

(18)Koga, S.; Williams, D. S.; Perriman, A.; Mann, S. Nat. Chem. 2o11, 3, 720 .

(19)Mathger, L. M.; Denton, E. J.; Marshall, N. J.; Hanlon, R. T. J. R.

Soc. Interface 2009, 6, S149.

(20)Richter, A.; Paschew, G.; Klatt, S.; Lienig, J.; Arndt, K. F.; Adler, H. J. P. Sensors 2008, 8, 561 .

(21)Philippova, O. E.; Hourdet, D.; Audebert, R.; Khokhlov, A. R.

Macromol.1997, 30, 8278.

(22)DeLuca, M.; McElroy, W. Biochemistry 1974, 13, 921.

(23)Ribeiro. C; Esteves da Silva. J. C. G. Photochem. Photobiol. Sci. 2008, 7,1085 .

(24)Melin, J.; Quake, S. R. Annu. Rev. Bioph. Biom. 2007, 36, 213. 
TOC graphic

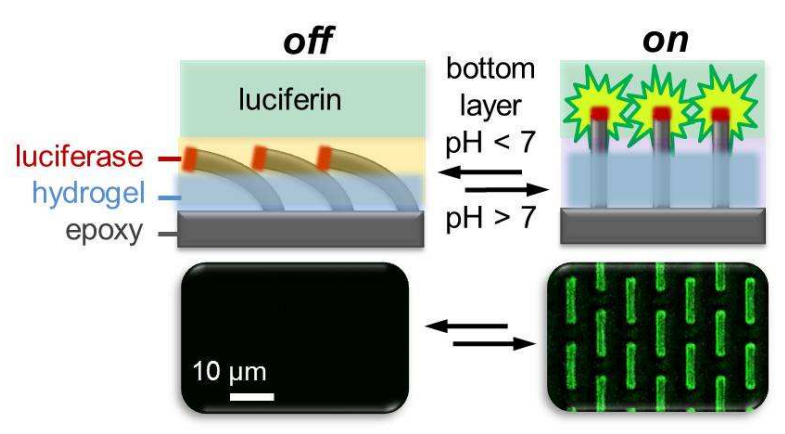

